\title{
Investigation of image segmentation methods for intracranial aneurysm haemodynamic research
}

\author{
Y. Sen, Y. Zhang, Y. Qian \& M. Morgan \\ Australian School of Advanced Medicine, \\ Macquarie University, Australia
}

\begin{abstract}
Patient-specific haemodynamic technology has been applied in clinical applications. Computational haemodynamic simulation is performed by utilization of geometric results obtained via medical image segmentation. However, the geometry and volume of intracranial aneurysm models are highly dependent upon different segmentation methods, even when employed upon the same medical imaging data. Moreover, methods of vascular segmentation have been insufficiently validated. In this study, we compared three segmentation methods; the Region Growing Threshold (RGT), Chan-Vese model (CV) and Threshold-Based Level Set (TLS), to segment the aneurysm geometry through the use of CTA image data. The results were evaluated via measurement of arterial volume differences (VD), local geometric shapes, and haemodynamic simulation results. We found that the maximum VD of three segmentation methods sat at around $\pm 15 \%$. Local artery anatomical shapes of aneurysms were likewise found to significantly influence segmentation results. The computational haemodynamic simulation was performed modelling three types of geometries, with typical haemodynamic characteristics; i.e. energy loss and shear stress. We found that there was a maximum of 58\% difference between segmentation methods. The results indicated that it is essential to validate segmentation methods in order to confirm the quality of segmentation processes in the application of patient-specific cerebrovascular haemodynamic analysis. Keywords: medical image segmentation, intracranial aneurysm, level set, region growing threshold, haemodynamic.
\end{abstract}




\section{Introduction}

Computed Tomography Angiography (CTA) is widely used in neurovascular imaging as a non-invasive diagnostic tool for detecting and evaluating intracranial aneurysms. This makes it possible to visualize three dimensional (3D) cerebral aneurysms, allowing us to reconstruct patient specific vessels. Currently, the 3D geometry blood vessel has been applied to perform haemodynamic simulations. The results have been applied as an intelligent diagnosis tool to support aneurysmal treatment. Computational fluid dynamics (CFD) technology has been applied to calculate blood pressure, velocity, wall shear stress (WSS), and energy loss (EL) [1]. These parameters are difficult to obtain via direct measurements. These computational haemodynamic parameters have provided useful information in support of vascular surgeons in disease diagnoses and surgical preparation.

The accuracy of CFD simulation is highly dependent on vessel geometry [2]. The methods of segmentation of medical images directly influence the accuracy of aneurysm model construction, especially in the shapes and volumes of the vessel. Despite the many image segmentation methods, each with varying approaches and algorithms, there are currently no dominant segmentation methods, in terms of effectiveness, across all areas [3-5]. Our previous study has indicated that the volume of the aneurysm models exhibit variances across different segmentation methods, with the segmentation method likewise influencing the local geometric shapes of the aneurysms [6]. Validation will thus be an essential process, comparing segmentation methods and adjusting the parameters of these segmentation techniques in order to confirm the quality of patient-specific cerebral-vascular haemodynamic analysis. Although a number of commercial segmentation software packages have been released to the market, there is a conspicuous lack of methodological discussion and information regarding validation processes.

In this paper, six CTA image data, which include vascular and cerebral aneurysm images, are employed. Three segmentation methods; the Region Growing Threshold (RGT), Chan-Vese model (CV) [7], and Threshold-Based Level Set (TLS) are applied to segment these image data. We compared the difference, calculated from the above three segmentation methods, and performed CFD simulation in order to compare its influence upon haemodynamic research.

\section{Materials and methods}

All the experiments were performed on cropped data sets to reduce calculation time and memory usage. A region of interest (ROI), a good representation of the targeted aneurysm region for segmentation, is selected depending on the aneurysm size. All preparatory work is carried out prior to conduction of the experiments. 


\subsection{Region Growing Threshold connecting (RGT)}

The Region Growing Threshold method begins from a seed(s) which is selected within the area of the object to be segmented. This method requires two intensity values for the pixel of the object, with both a low threshold $T_{1}$ and high threshold $T_{2}$ value. Neighbouring pixels, whose intensity values fall inside this range are accepted and included in the region. When no more neighbouring pixels are found that satisfy the criterion, the segmentation is considered to have finished. The selection criterion is described by the following equation:

$$
I(X) \in\left[X-T_{1}, X+T_{2}\right]
$$

where $T_{1}$ and $T_{2}$ represent the low and high thresholds of the region intensities, $I(X)$ represents the image and $X$ the position of the particular neighbouring pixel being considered for inclusion in the region. Problems of RGT include threshold selection and sensitivity to seed position [8].

\subsection{Chan-Vese model (CV)}

The Chan-Vese model is based on the Mumford and Shah [9] functional. The associated evolution PDE in the level set framework is represented as:

$$
\frac{\partial \varphi}{\partial t}=|\nabla \varphi|\left[\lambda_{2}\left(I-\mu_{\text {out }}\right)^{2}-\lambda_{1}\left(I-\mu_{\text {in }}\right)^{2}-\alpha+\beta \operatorname{div}\left(\frac{\nabla \varphi}{|\nabla \varphi|}\right)\right]
$$

where $\mu_{\text {in }}$ is the mean of the target object of intensity, $\mu_{\text {out }}$ represents the mean of the background of intensity and $\lambda_{1}, \lambda_{2} \alpha, \beta$ are positive constants. In the Chan-Vese model, there is no utilization of a term related to the image gradient. Instead, it utilizes region intensity information for the target objects of segmentation. This model has exhibited a significantly effective performance in segmentation of images with unclear boundaries.

\subsection{Threshold-based level set (TLS)}

The threshold-based level set combines the geodesic active contour [10] and the Chan-Vese model together within the level set framework.

Under the level set scheme, the contour deforms by the function; $\frac{\partial \Gamma(t)}{\partial t}+$ $F|\nabla \varphi|=0$, with an embedded surface $\Gamma(t)$ represented as the zero level set of $\varphi$ by $\Gamma(t)=\{x, y \in R \mid \varphi(x, y, t)=0\}$. $F$ is a function for speed, which drives the $\Gamma(t)$ surface evolution in the normal direction. It is clear that $F$ directly impacts upon the quality of medical image segmentation. The associated evolution PDE in the level set framework is as follows:

$$
\frac{\partial \varphi}{\partial t}=|\nabla \varphi|\left(\alpha(I-T)+\beta \operatorname{div}\left(g \frac{\nabla \varphi}{|\nabla \varphi|}\right)\right)
$$

where $I$ represents the image to be segmented, $T$ represents the intensity threshold, $g$ represents the image gradient, $\kappa=\operatorname{div}\left(\frac{\nabla \varphi}{|\nabla \varphi|}\right)$ represents the curvature, $\alpha$ represents the image propagation constant and $\beta$ represents 
the spatial modifier constant for the curvature $\kappa . \alpha$ and $\beta$ weight the relative influence of each of these terms on the movement of the surface contour.

The threshold-based level set requires an appropriate estimate of the threshold from proper segmentation of the aneurysm, obtained via Chan-Vese model segmentation and the concepts of both confidence interval (CI) and confidence level (CL).

According to the theory of confidence interval, the lower bound threshold of the aneurysm can be defined by:

$$
T_{i}=\mu_{a}-k_{i} \sigma_{a} \quad i \geq 0
$$

The threshold $\mathrm{T}$ is the difference between the mean of the contour of the aneurysm $\left(\mu_{a}\right)$ and $k$ times its standard deviation $\left(\sigma_{a}\right)$. The intensities of the aneurysm and its background regions are different. The lowest intensity threshold of the aneurysm is the same as the highest intensity threshold of the background. Thus, $\mu_{b}+k_{b} \sigma_{b}=\mu_{a}-k_{a} \sigma_{a}$ would apply. The confidence level for both the aneurysm and background is considered to be the same; $k_{b}=k_{a}=$ $k$. Therefore, $k$ can be described as:

$$
k=\frac{\mu_{a}-\mu_{b}}{\sigma_{a}-\sigma_{b}}
$$

We utilized the Chan-Vese model method to perform an initial segmentation. From the segmentation results, the initial $k_{0}$ can be calculated by eqn.(8). The initial $T_{0}$ can subsequently be found using equation (7).

\subsection{Data acquisition}

Clinical studies were performed with the consent of the patient in relation to aneurysm image acquisition. These protocols were approved by the local institutional review board and the regional research ethics committee. Six data sets of patients with internal carotid artery aneurysms were acquired by 3D CTA scans (GE Healthcare).

Cross-sectional images were acquired by a CT angiography scanner with multidetector-row capability, a table speed of $9 \mathrm{~mm} / \mathrm{s}$ and zero-degree table (and gantry tilt). Scanning started from the common carotid artery (CCA) and continued parallel to the orbito-meatal line to the level of the Circle of Willis, during which intravenous injection of contrast material was administered at a rate of $3.5 \mathrm{mls} / \mathrm{s}$. Aneurysm image was $512 \times 512$ pixel field, whilst continuous thickness slices were used to segment and reconstruct 3D vascular geometry. Pixels are expressed in Hounsfield Units (HU).

\subsection{Haemodynamic analysis}

CFD simulation includes three stages: 1) the segmentation of an aneurysm from medical imaging data recorded in DICOM format and generation of a STL format geometry data; 2) mesh generation; 3) CFD analysis. In the first stage, the segmentation methods mentioned above were introduced to reconstructive vessel and aneurysm geometry. In the second stage, ANSYS ICEM was used to form a 
mesh for CFD simulation. In the third stage, the geometric models are transferred to a haemodynamic system for CFD haemodynamic analysis.

\subsubsection{Mesh generation}

As the accuracy of CFD results depends upon grid resolution and boundary conditions, a series of analyses of verification and validation of mesh independent tests have been carried out at the beginning of this study. When the mesh number is found to be around at 400,000, the haemodynamic character; energy loss (EL), began to converge into a constant. Therefore, accurately reliable results could be obtained with a total of 563,000 finite elements and 240,000 nodes used in this study. In order to accurately calculate the WSS, five layers of prism mesh were inserted onto the artery's internal surface. The distance of the first prism mesh to the artery surface was set to $0.01 \mathrm{~mm}$.

\subsubsection{Calculation}

The flow simulation is based on the Navier-Stokes $(\mathrm{N}-\mathrm{S})$ momentum equation and continuity equation as defined below:

$$
\left\{\begin{array}{l}
\frac{\partial}{\partial t}\left(\rho u_{i}\right)+\frac{\partial}{\partial x_{j}}\left(\rho u_{i} u_{j}\right)=-\frac{\partial p}{\partial x_{i}}+\frac{\partial}{\partial x_{j}}\left[\mu\left(\frac{\partial u_{i}}{\partial x_{j}}+\frac{\partial u_{j}}{\partial x_{i}}\right)\right] \\
\frac{\partial \rho}{\partial t}+\frac{\partial}{\partial x_{j}}\left(\rho u_{j}\right)=0
\end{array}\right.
$$

where $i, j=1,2,3, x_{1}, x_{2}, x_{3}$ represents coordinate axes, $u_{\mathrm{i}}, u_{\mathrm{j}}$ and $p$ are the velocity vectors and the pressure in the point of the fluid domain, $\rho$ and $\mu$ are blood density and viscosity, $t$ is time.

\subsubsection{Energy loss}

Energy loss is calculated as the power difference from inlet to outlet, and can be calculated as follows:

$$
E L=\sum\left(P_{i}+\rho \frac{1}{2} v_{i}^{2}\right)-\sum\left(P_{o}+\rho \frac{1}{2} v_{o}^{2}\right)
$$

where $\rho$ is density, $v$ is velocity, $i$ indicates inlet, and $o$ means outlet.

\subsubsection{Wall shear stress (WSS)}

The wall shear stress (WSS) is derived from predicted flow velocities. The parameter is known to be associated with initial thickening and thrombosis formation. The WSS is defined as:

$$
W S S=-\left.\mu \frac{\partial v_{t}}{\partial n}\right|_{w a l l}
$$

where $\mu$ is the dynamic viscosity, $v$ is the velocity parallel to the wall and is the unit vector perpendicular to the wall. 


\section{Results}

\subsection{Geometric shapes}

Fig. 1(d) is a picture which is taken during aneurysm treatment for Case 1. Two protrusions; A and B, are clearly observed in the aneurysm, with protrusion A located upon the larger protrusion B. Fig. 1(a) and (c) indicate that the results of RGT and TLS methods segment both A and B protrusions. On the other hand, however, analysis of Fig. 1(b) reveals that the CV method leads to the display of only protrusion $\mathrm{B}$, whilst protrusion $\mathrm{A}$ is not replicated in the segmented result.

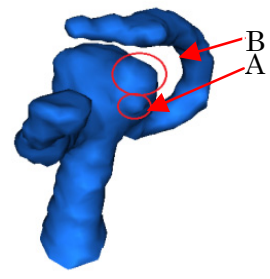

(a)

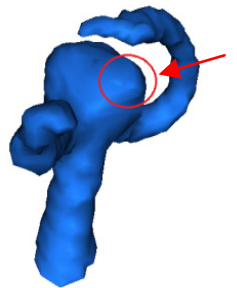

(b)

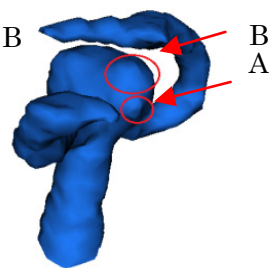

(c)

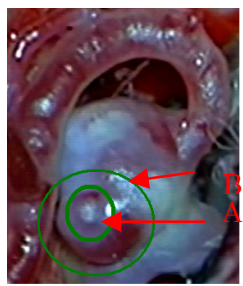

(d)

Figure 1: Geometric shape of aneurysm in Case 1. (a) RGT; (b) CV; (c) TLS; (d) surgical imagery.

\subsection{Model volumes}

All model volumes are listed in Fig. 2. The largest difference in terms of aneurysm volume, compared with TLS method, is around $\pm 15 \%$, as was the case for Case 4 . The difference across other cases was around \pm 5 to $\pm 10 \%$. The study indicates that the volume differences seen in aneurysm size may create an error of around $15 \%$, through the use of various segmentation methods.

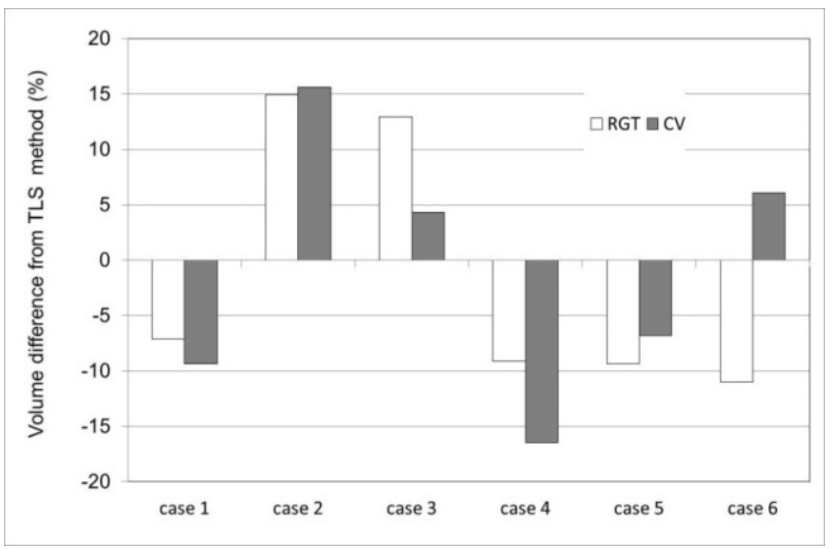

Figure 2: $\quad$ Aneurysm volume difference compared with TLS method. 


\subsection{Haemodynamic simulation results}

CFD simulation is performed by a process that includes segmented geometries. In order to compare various models under the same flow condition; inflow is assumed at $125 \mathrm{ml} / \mathrm{min}$. The outflow condition is extended to a length distant enough to allow pressure recovery at the outlets. Fig. 3 illustrates the pressure distribution on the aneurysm surface (Case 1 and Case 4), with the pressure distributions being significantly different between the various segmentation methods. Fig. 4 shows the results of EL, which were calculated based upon the three types of segmentation methods. In particular, for Case 4, there is a $58 \%$ difference when compared to the results of TLS segmentation method. The results indicate that the extensive dependence of haemodynamic results on the accuracy of segmentation methods. Fig. 5 depicts the distribution of WSS between Case 1 and Case 4. The results indicate that local differences arose for WSS, particularly in bleb areas.

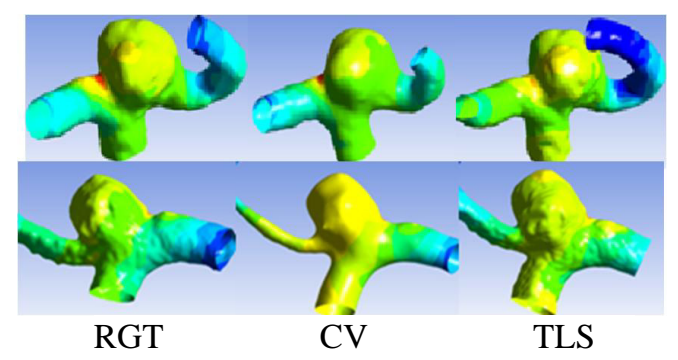

Figure 3: $\quad$ Pressure distribution: top line; Case 1, bottom line; Case 4.

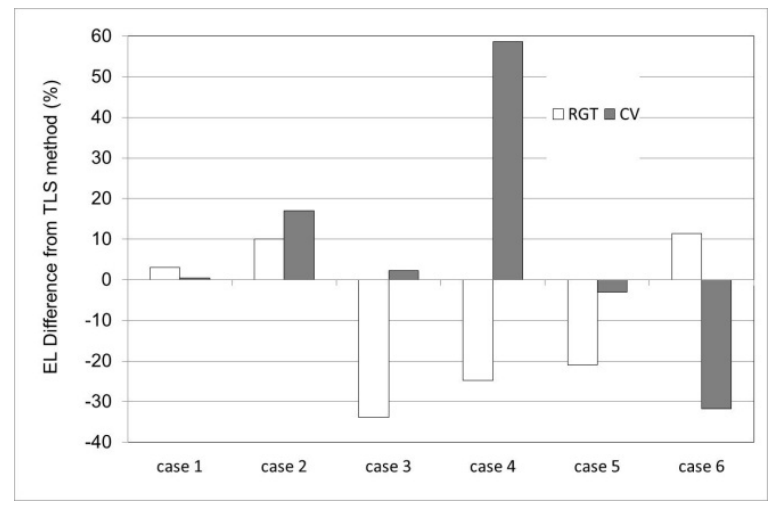

Figure 4: Haemodynamic results difference compared with TLS method. 


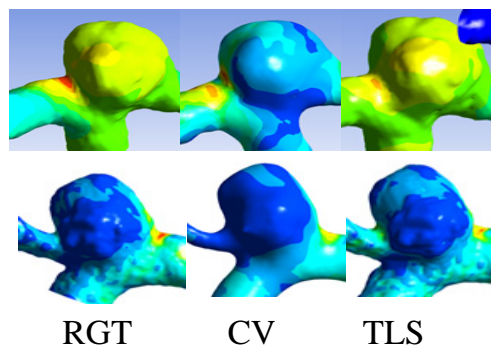

Figure 5: $\quad$ WSS distribution: top line; Case 1, bottom line; Case 4.

\section{Conclusions}

The study analysed six cerebral aneurysm models in geometric shape, volume and haemodynamic results. Although based on the same medical image data, different image segmentation methods generate alternations in shape, volume, thereby bringing significant haemodynamic results. The occurrence of uncertain results will negatively affect the accuracy of patient-specific haemodynamic applications.

The TLS method was proposed to improve cerebrovascular aneurysm segmentation applications, a technique with the ability to segment aneurysms automatically without the setting of a seed point or intensity threshold, which is likewise available for the segmentation of complex cerebrovascular anatomical shapes.

We believe a validation process to confirm segmentation will be an important study to limit the error arising from image segmentation. Results that are influenced by the segmentation process must be counted into the haemodynamic study. A series of in-vitro and in-vivo validation will thereby be performed in our projects.

\section{Acknowledgements}

The authors would like to thank the members of Macquarie Medical Imaging, Macquarie University Hospital, for their kind support and contribution on these cases.

\section{References}

[1] Y. Qian, et al. Risk Analysis of Unruptured Aneurysms Using Computed Fluid Dynamics Technology: Preliminary Results, AJNR Am J Neuroradiol. Nov-Dec 2011; 32(10):1948-55.

[2] Thomas, J.B., et al. Reproducibility of Image-Based Computational Fluid Dynamics Models of the Human Carotid Bifurcation. Annals of Biomedical Engineering, 2003. 31(2): p. 132-141. 
[3] Zuva, T., et al. Image Segmentation, Available Techniques, Developments and Open Issues. Canadian Journal on Image Processing and Computer Vision, 2011. Vol. 2 No. 3: p. 20-29.

[4] Zhang, H., J.E. Fritts, and S.A. Goldman, Image segmentation evaluation: A survey of unsupervised methods. Computer Vision and Image Understanding, 2008. 110(2): p. 260-280.

[5] Lesage, D., et al. A review of 3D vessel lumen segmentation techniques: models, features and extraction schemes. Medical image analysis, 2009. 13(6): p. 819-45.

[6] Sen, Y., et al. A Comparison of Medical Image Segmentation Methods for Cerebral Aneurysm Computational Haemodynamics, in 2011 4th International Conference on Biomedical Engineering and Informatics (BMEI) 2011. p. 901-904.

[7] Chan, T.F. and L.A. Vese, Active Contours Without Edges. IEEE Transactions on Image Processing 2001. vol. 10, no. 2: p. 266-277.

[8] Hu, G.a.M., Survey of Recent Volumetric Medical Image Segmentation Techniques, in Biomedical Engineering, C.A.B.d. Mello, Editor 2009, InTech.

[9] Mumford, D.B. and J. Shah, Optimal Approximations by Piecewise Smooth Functions and Associated Variational Problems. Communications on Pure and Applied Mathematics, 1989. 42(5): p. 577-685.

[10] Caselles, V., R. Kimmel, and G. Sapiro, Geodesic Active Contours. International Journal of Computer Vision, 1997. 22(1): p. 61-79. 